\title{
JAK2 inhibitor restores balance in polycythaemia vera
}

Polycythaemia vera (PV) is a myeloproliferative disease associated with a particular mutation in the tyrosine kinase JAK2, which is present in almost all patients with PV. The same mutation - JAK2V617F - can also be detected in about half the patients with the related diseases essential thrombocythaemia (ET) and myelofibrosis, making the JAK2 kinase an excellent target for therapeutic intervention. Now, two papers in Cancer Cell report a highly selective orally available JAK2 inhibitor, which shows promising properties in preclinical models of PV.

Myeloproliferative disorders (MPDs) are clonal haematopoietic disorders in which a hypersensitivity of haematopoietic progenitor cells (HPCs) to growth factors results in an overproduction of terminally differentiated cells. Patients are at risk of major thrombotic events and bleeding complications (due to the overproduction of erythrocytes in PV, and thrombocytes in ET), and risk progressing to acute myelogenous leukaemia. PV and ET can also progress to myelofibrosis, in which the bone marrow is replaced by scar tissue, commonly leading to death within 12 months. The incidence of MPDs is about fivefold higher than the incidence of chronic myeloid leukaemia - the first leukaemia to be treated with a molecularly targeted inhibitor.

Now, Wernig and colleagues assessed the safety and efficacy of a new, highly specific ATP-competitive JAK2 inhibitor, TG101348, in a mouse model of JAK2V617Finduced PV. Oral treatment with
TG101348 induced a dose-dependent response, as evidenced by a reduction in erythroid and myeloid progenitors, a reduction/elimination of extramedullary haematopoiesis, and a normalization of splenic architecture. The differentiation of other cell lineages arising from HPCs, such as T cells, was not affected by the drug and there was evidence that treatment with TG101348 may even result in the reversion of myelofibrosis. The authors further established several experimental approaches that may be of value in measuring surrogate endpoints for response in a clinical-trial setting. These include the quantitative genomic assessment of the mutant allele burden by PCR and measuring the activation status of JAK2 signal transduction targets such as STAT5 by flow cytometric analysis.

Meanwhile, Geron and colleagues explored the molecular details of JAK2V617F inhibition. Using xenogeneic transplantation models, the authors showed that the propensity of human PV HPCs and JAK2V617F-transduced cord blood cells to differentiate along the erythroid lineage could be inhibited by TG101348. By contrast, the differentiation of HPCs with normal JAK2 was much less affected by the drug. Moreover, the authors established that JAK2V617F-expressing HPCs harboured higher levels of the erythroid transcription factor GATA1 and lower levels of transcription factors for other cell lineages. This imbalance could be reversed by TG101348. The drug inhibited phosphorylation of

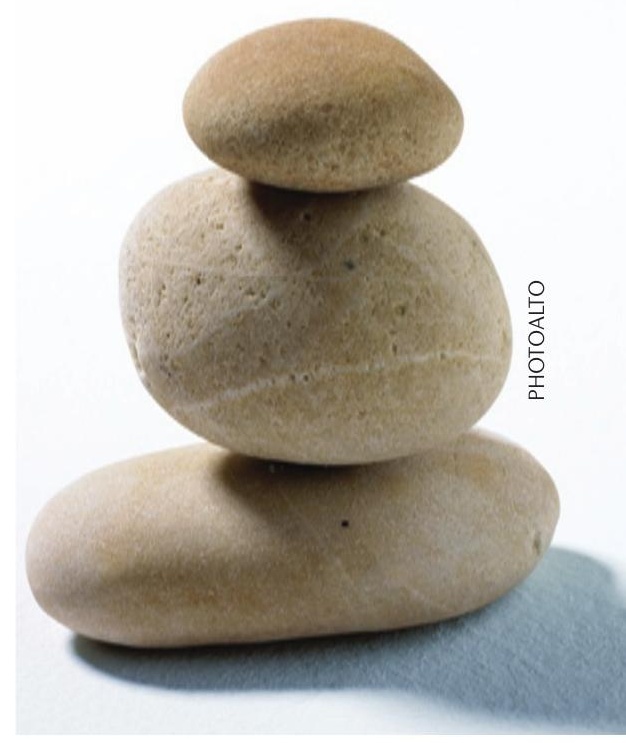

JAK2 downstream signalling factors such as STAT5 and AKT, as well as AKT-mediated GATA1 serine phosphorylation, resulting in inhibition of GATA1-mediated transcription of genes involved in erythroid differentiation.

Overall, there is a substantial MPD patient population that may benefit from a new therapeutic option. TG101348 is currently being evaluated in a Phase I clinical trial (NCT00631462), and data regarding safety are anticipated to be available next year. Importantly, the capacity of TG101348 to inhibit signalling through mutant JAK2 at the primitive progenitor level may be particularly relevant, as it was shown that progenitor cells can provide a reservoir for relapse for patients with chronic myeloid leukaemia treated with molecularly targeted drugs. Alexandra Flemming

ORIGINAL RESEARCH PAPERS Wernig, $\mathrm{C}$ et al. Efficacy of TG101348, a selective JAK2 inhibitor, in treatment of a murine model of JAK2V617F-induced polycythemia vera. Cancer Cell 13, 311-320 (2008) | Geron, I. et al. Selective inhibition of JAK2-driven erythroid differentiation of polycythemia vera progenitors. Cancer Cell 13, 321-330 (2008) 